\title{
TASKS OF SYSTEM IDENTIFICATION: A SIMPLIFIED APPROACH CREATED IN A DEVELOPING COUNTRY
}

\author{
Hesham W. Gomma \\ Electronics and Communication Department, \\ Faculty of Engineering, Helwan University, \\ Helwan, Egypt \\ E-mail:h_gomma@yahoo.co.uk
}

\begin{abstract}
This paper presents a simple identification algorithm for linear models. The main motivation behind this algorithm is to familiarize students and engineers having limited mathematical knowledge with the principles of system identification without adding more mathematical burdens. This is achieved by using the principles of the step response coefficients and its explicit relation with the system parameters. The new algorithm shows ability for identifying models with different orders based on using the physically tangible system step response. The efficiency of this algorithm is illustrated by simulations. Copyright (C) 2005 IFAC
\end{abstract}

Keywords: System Identification; Linear Systems; Impulse Responses; Step Responses; Education.

\section{INTRODUCTION}

Control engineering has significantly evolved during the last several decades, consequently its popularity has increased in developing countries and it has become an essential course in engineering schools. Nowadays this course receives as much attention as that given at European and American Universities. For example in Egypt, control is a compulsory course for students, of Electrical, Communication, Electronics, Computer, Biomedical and Mechanical Engineering, who supposedly have a considerable knowledge of mathematics. Unfortunately, especially in developing countries, standard Math courses have been perceived by the students as pure theoretical ones, thereby not the aim of Engineering schools. Therefore, by the time students are required to use it as an essential engineer's tool, it may be found that their background has been negatively affected by what can be termed 'mathematics contamination'. This ends up by students paying no attention to advanced control courses, which affects their ability to perform control engineering projects and to become modern practicing control engineers. One of the most crucial issues in control engineering dynamic process modelling and identifying system parameters to meet the requirements of the intended application - has been considerably affected. It has been noticed that most students and engineers face difficulties while modelling real systems, due to the fact that physical principles are not enough or too complicated to model the complete system. Therefore, the models may be identified using the conventional identification techniques such as generalized least-squares method, recursive least squares (Ljung, 1999), the subspace identification method (Peternell, 1996) and (Overschee, 1996), and recent techniques such as Ant system algorithm (Lei et al, 2001). Despite their attractive results, most of the existing techniques are rather complex or require special conditions to be used. Accordingly, the majority of students/engineers resort to use prewritten functions in MATLAB (or other software packages) to perform their identification tasks without understanding the theory of these techniques. Thus, they loose interest in using control engineering (classic or modern). Alternatively, they use other methods in controlling processes like writing linguistics programs with mostly poor performance.

This paper presents a technique for system identification that would help engineers and students in identifying complex systems by using 
conventional mathematics/system-analysis topics such as least square method and physical system step response. The proposed algorithm gives students/engineers an understating of the basic idea of system identification. In addition, it will encourage them to solve their own problems without using pre-written functions. Simulation results show that the proposed algorithm can give significant results.

\section{SYSTEM MODELING}

Most processes in industry when considering small changes around an operating point can be described by a linear model. One of the most crucial issues in modeling is to define the structure of the model. In this section, the following form of discrete time plant transfer function $F(z)$ will be considered while the order of the system will be selected in section 3

$$
F(z)=\frac{y(z)}{u(z)}=\frac{p_{0}+p_{1} z^{-1}+p_{2} z^{-2} \cdots p_{n} z^{-n}}{q_{0}+q_{1} z^{-1}+q_{2} z^{-2} \cdots q_{n} z^{-n}}
$$

where $y(t)$ and $u(t)$ are the system output and input respectively and $n$ is the numerator/denominator order. Executing synthetic division of the numerator by the denominator can lead to

$$
F(z)=\frac{y(z)}{u(z)}=f(0 T)+f(1 T) z^{-1}+f(2 T) z^{-2}+\cdots
$$

where

$$
\begin{aligned}
& p_{0}=f(0 T) q_{o} \\
& p_{1}=f(1 T) q_{o}+f(2 T) q_{1} \\
& \vdots \quad \vdots \quad \vdots \\
& p_{n}=f(n T) q_{o}+f(n-1) T q_{1}+f(n-2) T q_{2}+\cdots+f(0 T) q_{n}
\end{aligned}
$$

It is clear that $F(z)$ is represented in terms of the coefficients $f(0 T), \cdots, f(n T)$ (Jury, 1964). On the other hand, the known impulse response model (Camacho et al, 1995) for the same model of Equation (1) can be written as

$$
\begin{aligned}
y(t) & =\sum_{i=1}^{\infty} h_{i} u(t-i) \\
& =\left(h_{1}+h_{2} z^{-1}+h_{3} z^{-2}+\cdots\right) u(t)
\end{aligned}
$$

where $h_{1}, h_{2}, h_{3} \ldots$ are called the impulse response coefficients. Assume that the $u(z)$ is an impulse input then compare Equation (2) with (4), it can be seen that for the given transfer function, the coefficients of the quotient series are the impulse response coefficients. Therefore,

$$
\begin{aligned}
& h_{1}=f(0 T) \\
& h_{2}=f(1 T) \\
& \vdots \\
& h_{n}=f((N-1) T)
\end{aligned}
$$

which in turn has the following relation with the step coefficients

$$
\begin{aligned}
& g_{1}=h_{1} \\
& g_{2}=h_{1}+h_{2} \\
& g_{3}=h_{1}+h_{2}+h_{3} \\
& \vdots \\
& g_{n}=h_{1}+h_{2}+\cdots+h_{n}
\end{aligned}
$$

where $g_{i}$ stands for step response coefficients. Therefore, whenever a step response is available, step response and impulse response coefficients can be easily obtained. It should be noted that Equations (3) to (5) will be the key point in deriving the identification algorithm.

\section{IDENTIFICATION ALGORITHM}

In case of linear system identification, it is required to create a mathematical model which is able to provide a response that is similar to the one generated by the real (physical) system. The idea of the proposed algorithm is based on generating the step response of the system consequently the step response coefficients can be captured. Using equations (2) and (3), on the bases that impulse response coefficients are known and the system parameters are required to be identified, it can be found that it is required to solve a set of equations to estimate those parameters.

The first step after measuring the impulse response coefficients (from step response coefficients) is to substitute them in Equation (3), which then represents an explicit relation between the step response coefficients and system parameters. Consequently, a set of $n$-linear equations can be constructed, which can be written as follows

$$
\mathbf{A} \mathbf{x}=\mathbf{B}
$$

where

$$
\begin{aligned}
& \mathbf{A}=\left[\begin{array}{cccccccccc}
-1 & 0 & 0 & \cdots & 0 & f(0 T) & 0 & 0 & 0 & 0 \\
0 & -1 & 0 & \cdots & 0 & f(1 T) & f(0 T) & 0 & 0 & 0 \\
0 & 0 & -1 & \cdots & 0 & f(2 T) & f(1 T) & f(0 T) & 0 & 0 \\
\vdots & \vdots & \vdots & \ddots & \vdots & \vdots & \vdots & \vdots & \vdots & \vdots \\
0 & 0 & 0 & \cdots & -1 & f((n-1) T) & f((n-2) T) & \cdots & f(1 T) & f(0 T)
\end{array}\right] \\
& \mathrm{X}=\left[\begin{array}{cccccccccc}
p_{1} & p_{2} & p_{3} & \cdots & p_{n} & q_{1} & q_{2} & q_{3} & \cdots & q_{n}
\end{array}\right]^{T}
\end{aligned}
$$




$$
\mathbf{B}=\left[\begin{array}{c}
-f(1 T) q_{0} \\
-f(2 T) q_{0} \\
-f(3 T) q_{0} \\
\vdots \\
-f(n T) q_{0}
\end{array}\right]
$$

when the number of equations is larger than/(equal to) the number of unknowns this set can be solved using a suitable technique such as least squares. In least squares (Kreyszig, 1993), two variable $x$ and $y$ are related by $y=a+b x$ and a series of data have been measured to determine the two coefficients $a$ and $b$. This can be obtained by minimizing the following summation of

$$
q=\sum_{i=1}^{m}\left(y_{i}-a-b x_{i}\right)^{2}
$$

where $q$ depends on $a$ and $b$. In matrix form this can be written as

$$
q=\|\mathbf{y}-\mathbf{A} \mathbf{x}\|^{2}
$$

where $\|\cdots\|^{2}$ is the matrix 2-norm, and

$$
\mathbf{y}=\left[\begin{array}{c}
y_{1} \\
y_{2} \\
\vdots \\
y_{m}
\end{array}\right], \quad \mathbf{A}=\left[\begin{array}{cc}
1 & x_{1} \\
1 & x_{2} \\
\vdots & \vdots \\
1 & x_{m}
\end{array}\right], \quad \mathbf{x}=\left[\begin{array}{l}
a \\
b
\end{array}\right]
$$

This can be written as follows

$$
\begin{aligned}
q & =(\mathbf{y}-\mathbf{A x})^{T}(\mathbf{y}-\mathbf{A} \mathbf{x}) \\
& =\left(\mathbf{y}^{T}-\mathbf{x}^{T} \mathbf{A}^{T}\right)(\mathbf{y}-\mathbf{A} \mathbf{x}) \\
& =\mathbf{y}^{T} \mathbf{y}-\mathbf{y}^{T} \mathbf{A} \mathbf{x}-\mathbf{x}^{T} \mathbf{A}^{T} \mathbf{y}+\mathbf{x}^{T} \mathbf{A}^{T} \mathbf{A} \mathbf{x}
\end{aligned}
$$

Taking the derivative of equation (7) with respect to $\mathrm{x}^{T}$, then setting to zero, it can be found that

$$
\mathbf{x}=\left(\mathbf{A}^{T} \mathbf{A}\right)^{-1} \mathbf{A}^{T} \mathbf{y}
$$

Applying the same concept to equation (6), the system parameters can be easily obtained.

\section{SIMULATIONS RESULTS}

In this section, different simulation results are presented to show the ability of the new algorithm in giving good results. These examples introduce first, second and third order systems.

\section{Example 1}

Consider the following first order model

$$
\frac{y(z)}{u(z)}=\frac{0.4+0.6 z^{-1}}{1-0.8 z^{-1}}
$$

The aim here is to illustrate the ability of the new algorithm in identifying the system parameters. For simplicity, the response of the system to a unit step input (see Figure I) is determined, from which the impulse response coefficients can be obtained. Assuming a certain order for the identified system based on the experience of the designer, will allow the designer to decide the number of parameters that should be identified.

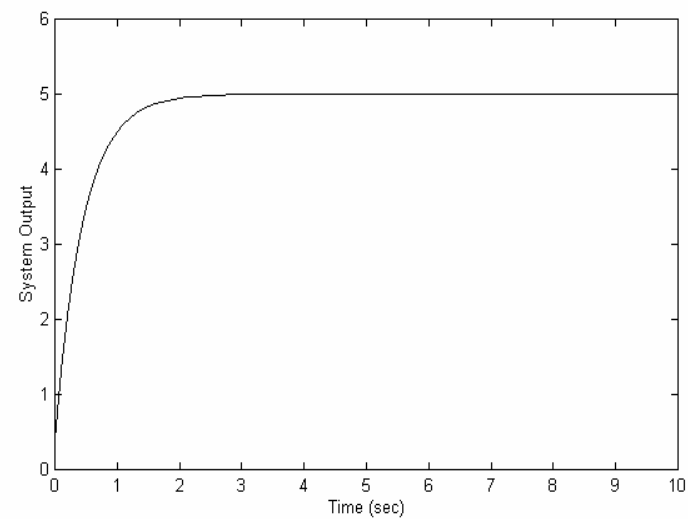

Fig. I: step response for first order model

Accordingly, most of designers can easily recognize that the response of Figure $\mathrm{I}$ is for a first order system, therefore, it is required to identify four parameters considering the following system

$$
\frac{y(z)}{u(z)}=\frac{p_{0}+p_{1} z^{-1}}{q_{0}+q_{1} z^{-1}}
$$

This can be achieved by constructing matrix A with dimension $(n \times 2)$ and vector $\mathrm{B}$ with dimension $(n \times 1)$ as shown in Equations (3) and (6). Then, using least squares will lead to the needed unknowns. They are found to be as follows

$$
q_{0}=1, q_{1}=-0.8, p_{0}=0.4, p_{1}=0.6
$$

which match the original model. In the mean time if another designer, suggests a higher order model for the above system, for example a second order with the following structure,

$$
\frac{y(z)}{u(z)}=\frac{p_{0}+p_{1} z^{-1}+p_{2} z^{-2}}{q_{0}+q_{1} z^{-1}+q_{2} z^{-2}}
$$

It can be seen that there are six parameters required to be identified. This can be achieved by constructing a matrix A with dimension $(n \times 3)$ and vector $\mathrm{B}$ with dimension $(n \times 1)$. It can be seen that after using least squares, the unknowns parameters are found to be

$$
\begin{aligned}
& q_{0}=1, q_{1}=-0.8, q_{2}=0, \\
& p_{0}=0.4, p_{1}=0.6, p_{2}=-2.595 \times 10^{-16} \approx 0
\end{aligned}
$$

It is clear that both results are almost identical which indicates the ability of the new algorithm in providing satisfactory results.

\section{Example 2}

In this example the following second order model is considered 


$$
\frac{y(z)}{u(z)}=\frac{0.0360 z^{2}+0.0326 z}{z^{2}-1.6722 z+0.7408}
$$

Applying the above algorithm considering a second order model

$$
\frac{y(z)}{u(z)}=\frac{p_{0}+p_{1} z^{-1}+p_{2} z^{-2}}{q_{0}+q_{1} z^{-1}+q_{2} z^{-2}}
$$

it is clear that there are six parameters should be identified. It is found that the algorithm gives exactly the same parameters

$$
\begin{aligned}
& q_{0}=1, q_{1}=-1.672, q_{2}=0.7408, \\
& p_{0}=0.03605, p_{1}=0.03261, p_{2}=8.607 \times 10^{-17} \approx 0
\end{aligned}
$$

Again, if it is suggested that the order of the system is a third order system, it can be easily seen that there are eight parameters to be identified and the suggested transfer function should take the following form

$$
\begin{aligned}
& \frac{y(z)}{u(z)}=\frac{p_{0}+p_{1} z^{-1}+p_{2} z^{-2}+p_{3} z^{-3}}{q_{0}+q_{1} z^{-1}+q_{2} z^{-2}+q_{3} z^{-3}} \\
& q_{0}=1, q_{1}=-0.3282, q_{2}=-1.507, q_{3}=0.995, \\
& p_{0}=0.03605, p_{1}=0.08106, p_{2}=0.04383, \\
& p_{3}=-4.197 \times 10^{-17} \approx 0
\end{aligned}
$$

It is clear that there is a difference between the estimated parameters (eight parameters) and the original ones (six parameters). Therefore, a comparison via simulation is shown in Figure II in which simulations are almost identical despite the fact that they are for two systems with different structures. This proves the ability of the proposed algorithm in system identification despite the suggested order.

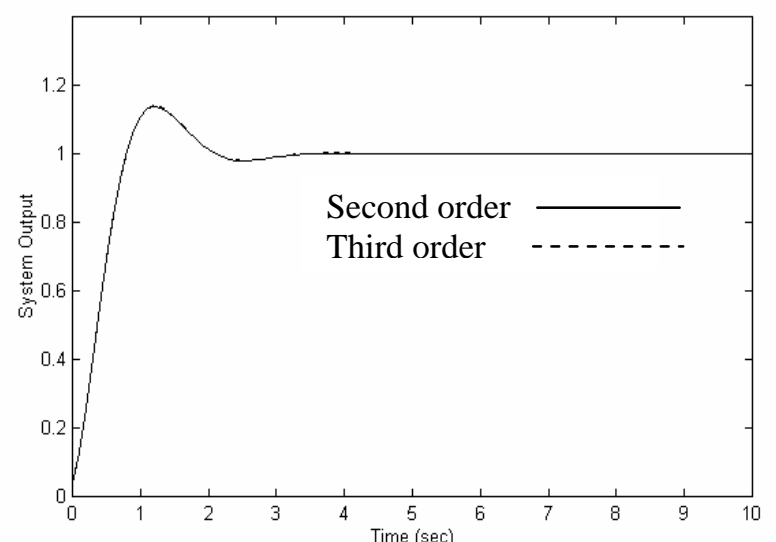

Fig. II: Step response for the original system (second order) and the identified (third order)

$$
\frac{y(z)}{u(z)}=\frac{0.007358 z^{3}-0.12325 z^{2}+0.049663 z}{z^{3}-2.2537 z^{2}+1.5986 z-0.33287}
$$

the system step response to unit step is shown in Figure III. Then, applying the above algorithm considering a third order model,

$$
\frac{y(z)}{u(z)}=\frac{p_{0}+p_{1} z^{-1}+p_{2} z^{-2}+p_{3} z^{-3}}{q_{0}+q_{1} z^{-1}+q_{2} z^{-2}+q_{3} z^{-3}}
$$

which means that there are eight parameters should be identified. It is found that

$$
\begin{aligned}
& q_{0}=1, q_{1}=-2.2537 q_{2}=-1.5986 q_{3}=-0.3328: \\
& p_{0}=0.07358,5 p_{1}=0.1232,5 p_{2}=0.049663 \\
& p_{3}=5.898 * 10^{17} \approx 0
\end{aligned}
$$

which are almost identical to the given system.

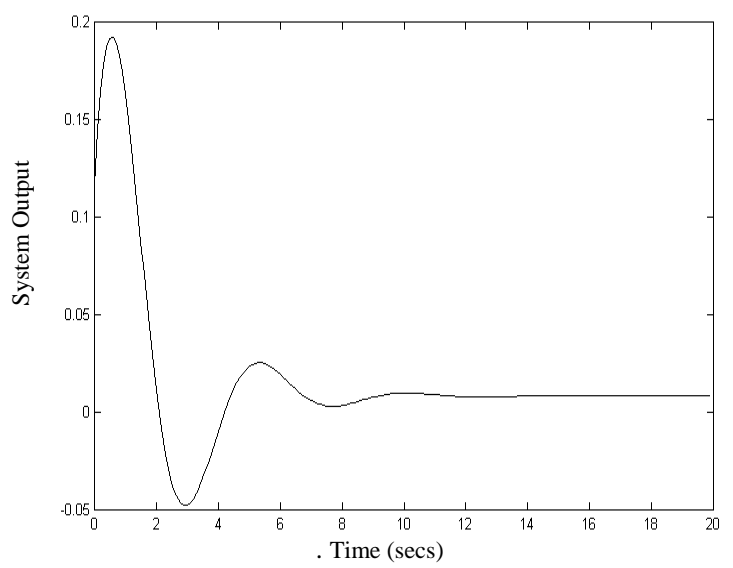

Fig. III: step response

Suggesting that the order of the system is fourth order system therefore a system in the following form will be considered

$$
\frac{y(z)}{u(z)}=\frac{p_{0}+p_{1} z^{-1}+p_{2} z^{-2}+p_{3} z^{-3}+p_{4} z^{-4}}{q_{0}+q_{1} z^{-1}+q_{2} z^{-2}+q_{3} z^{-3}+q_{4} z^{-4}}
$$

In this case there are ten parameters should be identified. Applying the algorithm has given the following values.

$$
\begin{aligned}
& q_{0}=1, q_{1}=-1.544 q_{2}=0, q_{3}=0.801 q_{4}=-0.23611 \\
& p_{0}=0.1163 p_{1}=-0.1514 p_{2}=-0.0237 p_{3}=0.0763 \\
& p_{4}=-0.01738
\end{aligned}
$$


It is clear that both simulations are almost identical despite their different orders. The simulations of both systems are shown in Figure IV.

This again shows the ability of the proposed algorithm in identifying system parameters and providing identical responses. This can be attributed to its concept in solving a set of equations in order to achieve a certain step response.

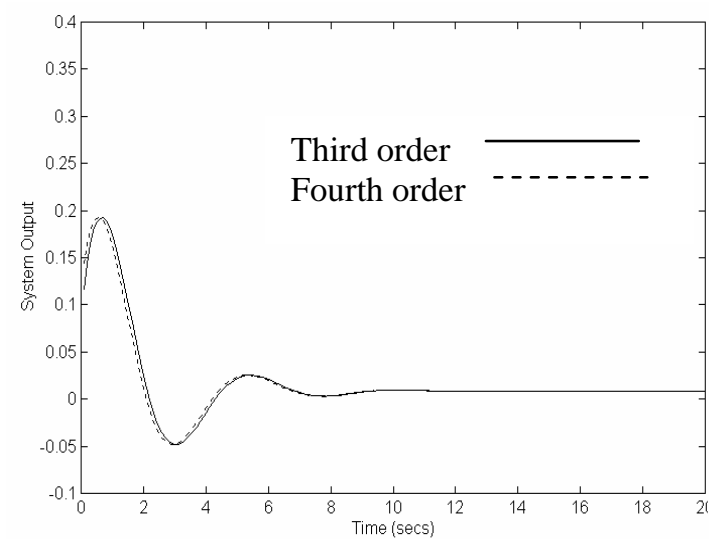

Fig. IV: Step response for the original system (third order) and the identified (fourth order)

\section{STUDENTS’ FEEDBACK}

The proposed algorithm has already been introduced to a sample of eighty six students in their final year of Electronics section at Helwan University, Egypt. It has been found that about $65 \%$ of the students are able to use the above algorithm in solving identification problems with clear ability in understanding the theoretical approach. Before introducing this algorithm, only $4 \%$ of the same sample of students managed to solve the same problems when they used the available Textbooks.

It is obvious that the proposed algorithm has a positive impact on the students' ability in identifying linear systems despite their orders or structures. This can be attributed to the simplicity of the algorithm. Therefore, this algorithm is strongly recommended to the aforementioned class of students and engineers who require identification techniques to help them in solving their design problems.

\section{CONCLUSION}

In this paper an identification approach has been introduced. The algorithm has shown ability in identifying different linear systems and providing similar responses despite the suggested orders. The main advantage, of the presented approach, is the use of the physically tangible system step response for identification. Moreover, this approach depends on simple mathematics in achieving its task therefore it is manageable for students/engineers with limited mathematical background especially in developing countries. In spite of the significant advances in the system identification field, this problem remains open to further avenues of research, however more attention should be given to educational purposes.

\section{REFERENCES}

Camacho, E.F. and C. Bodons, "Model predictive control in the process industry: Advances in industrial control”, Springer-Verlag, 1995.

Kreyszig, E., "Advanced engineering mathematics”, John Wiley \& Sons, 1993.

Lei, W., and W. Qidi, "Linear System Parameters Identification based on Ant System Algorithm", Proceeding of the 2001 IEEE International Conference on Control Applications, Mexico City, Mexico, pp. 401-406. 2001.

Ljung, L., "System Identification: theory for the user", Prentice-Hall Information and System Science Series, 1999.

Peternell, K., W. Scherrer and M. Deistler, "Statistical analysis of novel subspace identification methods”, Signal Processing, vol. 52, no. 2, pp. 161177, 1996.

Van Overschee, P., and B. De Moor, "Susbpace Identification for Linear Systems. Theory, Implementation and Applications", Dordrecht, The Netherlands: Kluwer Academic Publishers, 1996. 